\title{
TCOM AMÉRICA LATINA \\ Diez años de investigación de la comunicación pública de la ciencia en y desde América Latina. Un estudio en tres revistas académicas (2008-2017)
}

\section{Carlos Enrique Orozco}

Resumen

Palabras clave

DOI

Contexto: la comunicación pública de la ciencia
Las revistas académicas son uno de los principales medios que utilizan los científicos para dar a conocer los resultados de sus investigaciones, por lo que también pueden usarse como un registro de lo que se está investigando en un campo de conocimiento en particular como lo es la comunicación pública de la ciencia. En este trabajo presentamos las tendencias de la investigación académica en la comunicación de la ciencia en y desde América Latina tomando como corpus los reportes de investigación publicados en las tres principales revistas académicas internacionales en el campo: Science Communiation, Public Understanding of Science y Journal of Science Communicaton entre 2008 y 2017. Los hallazgos muestran un incremento de la producción, una clara hegemonía de Brasil, México y Argentina, los países económicamente más importantes de la región y una fuerte tendencia a la investigación que relaciona la comunicación de la ciencia con los medios de comunicación, en particular con la comunicación del riesgo medio ambiental en la región.

Comunicación académica; Divulgación de la ciencia en los países en desarrollo

https://doi.org/10.22323/3.01010202

Fecha de recepción: 13 de julio de 2018

Fecha de aceptación: 17 de octubre de 2018

Fecha de publicación: 21 de noviembre de 2018
Las prácticas de divulgación de la ciencia empezaron con los primeros científicos. Entre los primeros científicos que hacían divulgación se puede citar a Galileo porque además de sus investigaciones astronómicas se daba tiempo para publicar en italiano - y no en el latín habitual de la época - textos en formato de obra de teatro o demostraciones públicas de sus resultados en la torre de Pisa. Más reciente es el interés académico por estudiar estas prácticas. El antecedente más notable es el reporte titulado The Public Understanding of Science, realizado en 1985 por un equipo interdisciplinar en Gran Bretaña, mejor conocido como el Informe Bodmer. 
Una de sus recomendaciones fue la constitución del Comité de la Comprensión Pública de la Ciencia que estuvo formado inicialmente por un grupo de investigadores y profesionales alrededor del Science Museum de Londres que promovieron la creación de posgrados y publicaciones académicas especializadas en lo que se empezó a llamarse Public Understanding of Science y que en español se ha conocido por diversos nombres - divulgación, difusión, popularización, comunicación pública de la ciencia.

Considerando los públicos a los que va dirigido y especialmente la capacidad de éstos para descifrarlos, parto de la distinción que hace Antonio Pasquali entre difusión, divulgación y diseminación:

Se entiende por difusión el envío de mensajes elaborados en códigos o lenguajes universalmente comprensibles, a la totalidad del universo perceptor disponible en una unidad geográfica, cultural etc. Se entiende por divulgación el envío de mensajes elaborados mediante la transcodificación de lenguajes crípticos a lenguajes omnicomprensibles, a la totalidad del universo perceptor disponible. Se entiende por diseminación el envío de mensajes elaborados en lenguajes especializados, a perceptores selectivos y restringidos. [Pasquali, 1970, pp. 200, 201].

La comunicación de la ciencia, por definición, no es una actividad individual. Es un proceso que se entiende dentro de una dinámica social y política que tiene que ver con las complejas relaciones y articulaciones entre la ciencia, la cultura y la sociedad. Para abordar en todas sus dimensiones el tema tradicionalmente llamado de la divulgación científica, es necesario enfocar su estudio desde una perspectiva más amplia. Por lo anterior, en el texto de creación de la Maestría en Comunicación con especialidad en Difusión Científica y Cultural (1997) del ITESO, la Universidad Jesuita de Guadalajara (México), propusimos a la comunicación pública de la ciencia como "el conjunto de instituciones, estructuras mediadoras y prácticas sociales a través de las cuales se produce, circula y reproduce socialmente el sentido (conocimiento y disposición para la acción) con respecto al patrimonio científico y tecnológico de la sociedad." [Fuentes Navarro y Orozco, 1997].

En estos 30 años la comunicación pública de la ciencia ha pasado de ser el interés de unos cuantos a constituirse en una actividad académica que involucra a cientos de investigadores en los principales países del mundo.

Aunque no hay consenso entre los expertos sobre las condiciones que debe tener un objeto de estudio para que se convierta en un campo académico, se espera que cumpla con varias de las condiciones siguientes: un objeto de estudio claramente delimitado, una serie de conceptos básicos compartidos; una masa crítica de investigadores en activo, suficientes programas de investigación y docencia en universidades, cobertura internacional, comunidades académicas y profesionales visibles y organizadas, publicaciones especializadas y especialmente un cuerpo teórico que sustente la investigación empírica que se esté haciendo. La comunicación pública de la ciencia cumple con varios de las anteriores condiciones, pero no tiene un cuerpo teórico propio que fundamente la investigación que se hace desde diversos enfoques, por eso, abusando de la metáfora campirana, me atrevo a llamarla "parcela" de la comunicación pública de la ciencia [Trench y Bucchi, 2010]. 
Desde los tiempos de la Colonia, los primeros científicos en América Latina ya realizaban actividades científicas y tecnológicas de la época en áreas como la botánica, farmacoterapia, geografía, zoología, astronomía y matemáticas. Estos primeros científicos latinoamericanos en el siglo XVI, muchos de ellos (Fray Toribio de Motolinía, Fray Bernardino de Sahagún, entre otros) al amparo de conventos o abadías, se organizaban en sociedades científicas que difundían sus hallazgos y reflexiones en revistas y boletines como La Naturaleza, los Anales de Fomento o el Boletín de la Sociedad Mexicana de Geografía y Estadística. [Trabulse, 1983, p. 27]. En el siglo XIX y principios del XX, particularmente en los grandes países de la región como Brasil, México, Argentina y menor medida en Colombia, Perú y Venezuela, se fundaron instituciones públicas como zoológicos, parques botánicos, museos naturales, observatorios astronómicos para la divulgación del conocimiento, en particular, de la naturaleza. Sin embargo, no fue práctica generalizada ni tampoco objeto de política pública. El interés por parte de los poderes públicos de promover la ciencia y, también la divulgación de la misma se puede situar hasta mediados del siglo XX. En la ciudad de Caracas, la oficina Regional de Ciencia de la UNESCO para América Latina y el Caribe convocó en 1960 a la primera reunión de organismos gubernamentales en materia de ciencia y tecnología de países de América Latina para promover el desarrollo de políticas públicas en la materia. [Lemarchand, 2010]. Poco tiempo después en la "Declaración de Punta del Este" en 1967 los jefes de Estado de los países firmantes se comprometieron a crear organismos capaces de orientar, coordinar y desarrollar una política científica-tecnológica propia. En la presentación del documento, los presidentes dicen:

\footnotetext{
La ciencia y la tecnología son instrumentos de progreso para la América Latina y necesitan un impulso sin precedentes en esta hora. Este esfuerzo demanda la cooperación interamericana dada la magnitud de las inversiones requeridas y el nivel alcanzado por esos conocimientos. Del mismo modo, su organización y realización en cada nación no puede formularse al margen de una política científica y tecnológica debidamente planificada dentro del marco general del desarrollo. [OEA. Organización de Estados Americanos, 1967].
}

A partir de esa Declaración, los países con más peso económico en la región como Brasil, Argentina, México y Colombia crearon sus organismos de políticas pública para la ciencia, como fue el caso del Consejo Nacional de Ciencia y Tecnología (Conacyt) de México en 1970. En esos años y bajo la influencia de la experiencia estadounidense de la educación científica se empezó a discutir la pertinencia de que la ciencia y la tecnología estuvieran al alcance de todos los sectores sociales. Veinte años después - en 1990 - se fundó la Red de Popularización de la Ciencia y Tecnología para América Latina y el Caribe (Red POP), promovida por la Oficina Regional de Ciencias de la UNESCO para América Latina y el Caribe. [Massarani, Aguirre y col., 2015], que vendría a dar un fuerte impulso a las actividades de divulgación científica y tecnológica en los países de la región. Esta organización realiza congresos bienales, publica libros, organiza talleres de capacitación. Hasta la fecha es la principal organización dedicada a promover la comunicación de la ciencia en América Latina.

En los últimos 30 años, ha habido un impulso a estas actividades en la región, pero que son llamadas de diferente nombre. En el estudio Políticas públicas e instrumentos 
para el desarrollo de la cultura científica en América Latina, coordinado por Fernández Polpuch, Bello y Massarani [2016] dedican un espacio al tema de los varios nombres con los que se conocen estas actividades:

\begin{abstract}
En los países de América Latina se utilizan a menudo como sinónimos, distintos términos como: "popularización", "divulgación" y "comunicación" de la ciencia. "Apropiación social de la ciencia" y "desarrollo de la cultura científica" aparecen como conceptos estrechamente ligados, utilizados de manera creciente en los últimos años. "Periodismo científico", que de alguna manera es un concepto subordinado, también es utilizado, así como "difusión" y "promoción" que en realidad son algo ajeno al área específica. También se utiliza el término "Comunicación Pública de la Ciencia" y su adopción es apoyada por diversos expertos, especialmente de la Red Internacional de Comunicación Pública de la Ciencia y la Tecnología (PCST, del inglés Public Communication of Science and Technology), principal foro en la materia. En algunas regiones, como el Reino Unido y Estados Unidos, se utiliza con frecuencia también el término "public understanding of science" (comprensión pública de la ciencia) que se refiere al conjunto de factores relacionados con el interés, el conocimiento y las actividades que tienen al público sobre general sobre estas actividades [Fernández Polpuch, Bello y Massarani, 2016, p. 17].
\end{abstract}

En el libro titulado Aproximaciones a la investigación en divulgación de la ciencia desde América Latina a partir de sus artículos académicos [Massarani, Rocha y col., 2017] se dedica el segundo capítulo a los diversos términos utilizados por autores de América Latina en sus investigaciones con base a cuatro ejes de análisis: semántico, temporal, geográfico y de escenarios y áreas de trabajo, y llegaron a la conclusión siguiente:

\begin{abstract}
Mostramos que existe una relación entre los diferentes escenarios y los usos de los términos en ellos. También que éstos fueron cambiando según las décadas en las que los artículos analizados fueron producidos o el país desde el que los autores los escribieron. También según el área temática en el marco de la cual se realiza la divulgación científica. [Rocha, Massarani y Pedersoli en Massarani, Rocha y col., 2017, p. 55].
\end{abstract}

En un diagnóstico reciente sobre la divulgación de la ciencia en América Latina, los autores encontraron que es una actividad que se hace principalmente a través de los medios de comunicación masiva, aunque hay también actividades presenciales; que esta actividad está "aún muy lejos de ser sistemática y cotidiana en la vida de los latinoamericanos"; ha estado centrada en adolescentes y escolares, que no se disponen de suficientes recursos y en muchos casos de planes y evaluaciones formales y que una buena parte del personal que hace estas actividades no es profesional, sino que lo hace bajo la modalidad del voluntariado. [Patiño, Padilla y Massarani, 2017, pp. 121-127].

\title{
La producción académica sobre CPC en América Latina
}

La producción académica sobre comunicación pública de la ciencia se remonta a mediados de la década de los años 80 y ha habido algunos estudios mediante técnicas de bibliometría o estudios de análisis de contenido de las revistas académicas. En años recientes (2017) se publicó, un estudio de tendencias, a cargo 
de Guenther Lars y Marina Joubert. Estos autores revisaron la producción de investigaciones en comunicación pública de la ciencia publicadas en todos los números publicados hasta 2016 de las tres revistas más importantes en el campo: Science Communication, Public Understanding of Science y el Journal of Science Communication [Guenther y Joubert, 2017]. Ese mismo año se publicó, por la UNESCO y la Red POP, el primer estudio sobre estas tendencias en la región: Aproximaciones a la investigación en la divulgación de la ciencia en América Latina a partir de sus artículos académicos [Massarani, Rocha y col., 2017], con el objetivo de "identificar y analizar la producción académica en la región, desafío nada pequeño, considerando que la producción es, en gran medida, difusa: con pocas revistas científicas en divulgación de la ciencia y, por otro lado, por ser un campo interdisciplinario, la producción se distribuye en una serie de revistas de campos diferentes, de países diferentes". [Massarani, Rocha y col., 2017, p. 10]. Los autores trabajaron con un corpus de 609 artículos publicados en 80 revistas científicas.

Los resultados muestran, entre otros aspectos, una concentración de las publicaciones provenientes de Brasil, seguido por Colombia, Argentina y México. Los medios masivos de comunicación fueron el tema que más generó artículos en nuestro corpus, con 188 de los artículos (equivalente a 31\%), seguido por los museos de ciencia y de la relación entre divulgación de la ciencia y el ambiente escolar. Esperamos que estos resultados auxilien a entender la dinámica de la consolidación del campo académico de la divulgación de la ciencia en la región y estimulen la inversión en más estudios en este campo. [Massarani, Rocha y col., 2017, p. 13].

\section{Las revistas académicas en comunicación pública de la ciencia}

En la comunicación pública de la ciencia hay académicos laboriosos que están produciendo conocimiento en una buena cantidad de universidades en todo el mundo que se está diseminando por medio de las habituales reuniones académicas, pero principalmente en los journals. [Guenther y Joubert, 2017].

Las revistas académicas se pueden estudiar también como medios de comunicación que diseminan los conocimientos generados en universidades y centros de investigación, aunque estos estudios mediante técnicas bibliométricas tienen sus limitaciones. Por una parte, la publicación en revistas académicas indexadas son uno de los principales medios de legitimación de la producción de los investigadores, pero también es cierto que estos mecanismos están siendo criticados por servir más a la multiplicación de reportes para ganar nombre y evaluaciones favorables en los sistemas de reconocimiento - como el SNI de Conacyt en México, un sistema público que reconoce y otorga estímulos económicos a los investigadores que cubren una serie de requisitos, entre ellos publicar-, que en un verdadero medio para intercambiar resultados y propuestas académicas. Por otra parte, las revistas académicas tradicionales están perdiendo importancia por el empuje de las nuevas publicaciones de libre acceso, que algunas de ellas no forman parte de los índices reconocidos, pero que están generando nuevas formas de comunicación entre académicos. Sin embargo, a pesar de sus limitaciones, la publicación de investigaciones en revistas académicas indexadas es, junto con las memorias de los congresos y otras reuniones académicas, la principal de diseminación de los reportes de investigación en un campo disciplinar o en formación, como lo es el caso de la comunicación pública de la ciencia. [Guenther y Joubert, 2017]. 
Las revistas académicas son editadas por universidades, centros de investigación o empresas especializadas como Sage. Su estructura organizativa es muy similar: cuentan con un comité editorial formado por académicos de diversas instituciones; director y editor, quienes se apoyan en lectores externos, que no necesariamente forman parte del comité editorial, pero son reconocidos expertos en el campo y son quienes dictaminan, por lo general mediante mecanismo de "doble ciego", los artículos mandados para su publicación. Dado que el mundo académico existe la norma imperiosa del publish o perish, una revista académica consolidada no tiene problemas para conseguir artículos. El prestigio de la revista determina el número de artículos a revisión que reciben.

Los académicos que investigan en la comunicación pública de la ciencia también publican sus revistas especializadas o envían los resultados de sus investigaciones a journals cercanos en términos disciplinarios como los estudios sociales de la ciencia, la educación científica, comunicación o filosofía de la ciencia.

En el ya citado texto sobre la publicación de textos de comunicación pública de la ciencia en América Latina [Massarani, Rocha y col., 2017] se encontraron textos en 80 publicaciones distintas, aunque la inmensa mayoría en revistas brasileñas y publicadas solo en portugués.

Los journals internacionales especializados en comunicación de la ciencia son pocos y muy recientes. Los más reconocidos son Science Communication; Public Understanding of Science y el Journal of Science Communication. En el citado artículo de Lars y Joubert, los autores usaron las mismas revistas académicas para hacer su estudio de tendencia, debido a que son "las tres principales revistas académicas en el campo". [Guenther y Joubert, 2017, p. 2].

Science Communication: Linking theory and practice (SC) es la más antigua revista académica en esta área. Fue fundada en marzo de 1979. En sus primeros años estaba mucho más orientada a la sociología del conocimiento, pero con el tiempo se alejó de su orientación inicial y se fue centrando más en la comunicación de la ciencia. SC se define como:

\footnotetext{
una revista académica interdisciplinaria e internacional en ciencias sociales que examina la naturaleza del conocimiento experto (expertise), la difusión del conocimiento y la comunicación de los profesionales de la ciencia y la tecnología con el público. SC prioriza los aspectos teóricos y pragmáticos de las actuales debates sociales y políticos. Su orientación trasciende las fronteras nacionales, culturales y económicas en temáticas como las políticas de salud, las reformas educativas, el desarrollo internacional y los riesgos ambientales (http://scx.sagepub.com/).
}

Public Understanding of Science (PUS), fundada en 1992 por un grupo de interesados en la comunicación de la ciencia, entre ellos su primer editor, John Durant, del Science Museum de Londres. Es el journal con mayor reconocimiento en el área. Ocupó el lugar número 9 de 79 en el ranking de revistas académicas de comunicación en el 2016 y el primero de 44 en el campo de historia y filosofía de la ciencia. (http:/ /journals.sagepub.com/home/pus). PUS se define en su página web como una revista académica "internacional, totalmente arbitrada, que cubre todos los aspectos de las relaciones recíprocas entre la ciencia - incluyendo 
tecnología y medicina - y el público"

(http://pus.sagepub.com/content/by/year). Los principales temas que se publican son estudios de percepción y actitudes ante la ciencia y la tecnología; representaciones e imaginarios sobre la ciencia; creencias científicas y paracientíficas; historia de la ciencia; educación científica; la ciencia en los medios; ciencia ficción: museos y exhibiciones científicas; ciencia y el público, ciencia y tecnología en países en desarrollo y temas asociados.

Journal of Science Communication (JCOM) es la más reciente. Fue fundada en 2002 por el Programa en Comunicación de la Ciencia de la Scuola Internazionale di Studi Superiori Avanzati en Trieste, Italia. La editora actual es Emma Weitkamp de la Universidad de Bristol en la Gran Bretaña y cuenta con un comité asesor internacional formado por 26 miembros, de los cuales 2 son latinoamericanos. Se define como un journal de libre acceso en comunicación de la ciencia. Quiere aportar reflexiones teóricas y experiencias de trabajo tanto para los estudiosos como los practicantes del campo de la comunicación de la ciencia. La presentación de su página web dice:

\begin{abstract}
¿Por qué comunicación de la ciencia? Porque queremos cambiar y dialogar con el mundo de los estudios sociales de la ciencia, enfatizando la importante del proceso comunicativo en el desarrollo de la ciencia y la dinámica de las contemporáneas sociedades del conocimiento. (...) Queremos contribuir con la libre circulación de información y con perspectivas no eurocéntricas, con visiones heterogéneas como los estudios de género, la historia social, la investigación acción. Queremos que JCOM sea una plataforma por la que comunidades distantes (académicos, periodistas, científicos, museógrafos) pueden acercarse y dialogar. (...) A fin de cuentas, JCOM investiga las necesidades de comunicación entre la ciencia y los ciudadanos y dentro de las comunidades de científicos. (JCOM, http:/ /jcom.sissa.it).
\end{abstract}

Este artículo busca conocer las tendencias de la investigación de la comunicación pública de la ciencia en y desde América Latina tomando como corpus los artículos de investigación publicados en las tres revistas citadas en la década que va entre 2008 y 2017. Las tendencias se perfilan por medio de cuatro categorías: territorio o país, autoría, temas y subtemas y método utilizado.

Este artículo es un producto particular de la investigación "Tendencias del conocimiento en revistas académicas internacionales de la comunicación pública de la ciencia" en la que estoy analizando la producción académica en este objeto de conocimiento en los años recientes y que ha sido publicada en journals, con circulación - impresa o electrónica - internacional. Los criterios considerandos para las revistas son: a) que estén centradas en la comunicación pública de la ciencia, b) que sean revistas académicas con periodicidad regular, que cuenten con un comité editorial público y criterios editoriales explícitos, c) que tengan carácter internacional tanto su cuerpo editorial como sus autores, d) que cuenten con más de cinco años de existencia continua y e) que estén publicadas en inglés para que se tenga acceso desde variados países porque el inglés, por razones económicas, políticas y de hegemonía educativa, se ha convertido en la lengua franca en nuestro tiempo. Las tres revistas mencionadas cumplen con estas condiciones. 
El corpus está formado los artículos publicados entre 2008 y 2017 en la categoría de reportes de investigación; no estoy considerando editoriales, ni ensayos, comentarios o reseñas de libros. El texto considerado es la versión que aparece publicada en la página electrónica de la revista, con independencia de que no sea la primera versión enviada a la revista. La distribución de los 164 números de las revistas por año puede verse en la Tabla 1.

Tabla 1. Distribución de los números de las revistas.

\begin{tabular}{|c|c|c|c|r|}
\hline Año & $\begin{array}{c}\text { Science } \\
\text { Communication }\end{array}$ & $\begin{array}{c}\text { Public Understanding } \\
\text { of Science }\end{array}$ & $\begin{array}{c}\text { Journal of Science } \\
\text { Communication }\end{array}$ & Totales \\
\hline 2008 & 4 & 4 & 4 & 12 \\
2009 & 4 & 6 & 4 & 14 \\
2010 & 4 & 6 & 4 & 14 \\
2011 & 4 & 6 & 4 & 14 \\
2012 & 6 & 8 & 4 & 18 \\
2013 & 6 & 8 & 4 & 17 \\
2014 & 6 & 8 & 4 & 18 \\
2015 & 6 & 8 & 6 & 18 \\
2016 & 6 & 8 & 5 & 19 \\
2017 & 6 & 8 & $42 \quad(25.6 \%)$ & 164 \\
\hline Suma & $52 \quad(31.7 \%)$ & $70 \quad(42.7 \%)$ & & \\
\hline
\end{tabular}

Lo primero que puede advertirse claramente es que la tendencia es hacia una mayor publicación de número de las revistas y por tanto de artículos de investigación. En el caso de PUS se duplicó el número de revistas por año, al pasar de 4 en 2008 a 6 en 2009 y 8 a partir de 2012. En las otras dos revistas también se incrementaron los números por año, aunque en proporción menor; de 4 (2008) a 6 (2013) en el caso de SC y en JCOM pasó de 4 (2008) a 6 en 2016, aunque con una disminución en 2017. Esta tendencia a una mayor difusión y producción de investigaciones en comunicación pública de la ciencia también fue reportada por Lars y Joubert (2017).

En estos 164 números se publicaron en total 954 reportes de investigación. La distribución por revista y por año se puede ver en la Tabla 2.

En la Tabla 2 se confirma la afirmación anterior del incremento de artículos de investigación difundidos al año, al pasar de un total de 61 en 2008 a 113, casi el doble, en 2017.

Soy consciente de las dificultades conceptuales de agrupar con el término genérico de "América Latina" al conocimiento generado sobre esta parte del mundo en estos tiempos de globalización y máxime cuando se trata de textos difundidos en revistas en lengua inglesa, algunos producidos desde Estados Unidos o Italia. Sin embargo, América Latina es más que un conjunto de países en una zona geográfica determinada, sino una región económica y sociocultural en donde conviven varias lenguas y decenas de dialectos. Lo que en principio tenemos en común la mayor parte de las naciones de América Latina ${ }^{1}$ es nuestra herencia hispano-portuguesa,

\footnotetext{
${ }^{1}$ Excluyo los pequeños países caribeños con que fueron colonizados por los ingleses o franceses.
} 
Tabla 2. Distribución de los artículos de investigación por revista y por año.

\begin{tabular}{|c|r|r|r|r|}
\hline Año & $\begin{array}{c}\text { Science } \\
\text { Communication }\end{array}$ & $\begin{array}{c}\text { Public Understanding } \\
\text { of Science }\end{array}$ & $\begin{array}{c}\text { Journal of Science } \\
\text { Communication }\end{array}$ & Suma \\
\hline 2008 & 22 & 27 & 12 & 61 \\
2009 & 22 & 45 & 14 & 81 \\
2010 & 20 & 49 & 15 & 84 \\
2011 & 21 & 53 & 13 & 87 \\
2012 & 31 & 63 & 11 & 105 \\
2013 & 28 & 65 & 11 & 104 \\
2014 & 29 & 65 & 17 & 111 \\
2015 & 27 & 62 & 15 & 104 \\
2016 & 27 & 51 & 26 & 104 \\
2017 & 24 & 61 & 28 & 113 \\
\hline Suma & 251 & 541 & 162 & 954 \\
\hline Porcent. & $26.3 \%$ & $56.7 \%$ & $16.9 \%$ & $99.9 \%$ \\
\hline
\end{tabular}

pero que en la actualidad se manifiesta en relación de subordinación económica y tecno científica con Estados Unidos y algunas naciones europeas.

Investigar la comunicación de la ciencia en y sobre América Latina reconoce las tres manifestaciones de la comunicación pública de la ciencia que se incluyen en el corpus. Textos como los de Biro [2012], Ramalho, Polino y Massarani [2012] son ejemplos del conocimiento que sobre algún aspecto de nuestra región se están generando en nuestras universidades; mientras que autores como Gordon, Deines y Havice [2010] investigan, por ejemplo, la prensa de la ciudad de México, pero desde alguna universidad de Estados Unidos o también como Bruno Takahashi, peruano de origen y profesor de la Universidad del estado de Michigan, pero que investigó la cobertura del cambio climático en la prensa peruana [Takahashi, 2011].

Los indicadores para establecer las posibles tendencias de la producción de conocimiento en comunicación pública de la ciencia producido en y sobre América Latina tienen que ver con territorios o escenarios de estudio, autores, temas y métodos utilizados.

De los 954 artículos publicados entre 2008 y 2017 en las tres revistas, 40 (cifra que representa $4 \%$ del total) tienen como lugar de estudio empírico, algún país, grupo de naciones o región de América Latina. Este porcentaje es menor al de las naciones líderes de la globalización desigual. En el citado estudio de Lars y Joubert mencionan que los países angloparlantes son, con mucho, los principales lugres de donde provienen los artículos de investigación. "En este estudio, los Estados Unidos contabilizaron 427 [34.5\%] de los 1,237 artículos incluidos en el ejercicio de mapeo, mientras que el Reino Unido contabilizó 270 [21.8\%], seguido por Canadá [77 o $6.2 \%$ ] y Australia [51 o 4.1]. En conjunto estos cinco países contribuyeron con 825 artículos [66.7\%] del total". [Guenther y Joubert, 2017, p. 4].

La frecuencia de publicación de artículos de investigación en y sobre América Latina fue casi constante en los diez años de la muestra. El promedio fue de cuatro 
artículos por año, con un máximo de cinco y un mínimo de dos. Respecto a la distribución de artículos por revista; PUS publicó 17, mientras que JCOM 16 artículos en los diez años y SC solo 7, pero en términos relativos la que publicó más artículos fue JCOM porque esta revista publicó solo 162 artículos de investigación contra de 541 que publicó Public Understanding of Science en los mismos diez años. La proporción de artículos sobre América Latina sobre el total fue de 9.8 por ciento en JCOM; 3.1 por ciento en PUS y 2.7 por ciento en SC.

Tabla 3: Datos, autores, tema y territorio de los artículos del corpus.

\begin{tabular}{|c|c|c|c|c|}
\hline Revista & Año & Autores & Tema & Territorio \\
\hline JCOM & 2008 & $\begin{array}{l}\text { Veneu, F., Amorin, } \\
\text { L.H. y Massarani L. }\end{array}$ & $\begin{array}{l}\text { Periodismo de ciencia en América Lati- } \\
\text { na }\end{array}$ & $\begin{array}{l}\text { América } \\
\text { Latina }\end{array}$ \\
\hline JCOM & 2008 & Firer, M. & $\begin{array}{l}\text { Estudio de caso de museo de ciencias } \\
\text { ambulante }\end{array}$ & Brasil \\
\hline JCOM & 2008 & $\begin{array}{l}\text { Pioli A.L. y Da Costa, } \\
\text { M. C. }\end{array}$ & $\begin{array}{l}\text { Participación pública en la gestión del } \\
\text { agua en Brasil }\end{array}$ & Brasil \\
\hline JCOM & 2008 & $\begin{array}{l}\text { Lima M.T., Das Ne- } \\
\text { ves E.F. y Dagnino R. }\end{array}$ & Popularización de la ciencia en Brasil & Brasil \\
\hline SC & 2008 & Reis, R. & $\begin{array}{l}\text { Debate en periódicos sobre células ma- } \\
\text { dre en Brasil y Estados Unidos }\end{array}$ & $\begin{array}{l}\text { Brasil y } \\
\text { Estados Unidos }\end{array}$ \\
\hline JCOM & 2009 & $\begin{array}{l}\text { Franco Carvalho J. D. } \\
\text { y Buzá J. G. }\end{array}$ & $\begin{array}{l}\text { Comunicación de la ciencia en la en- } \\
\text { señanza de la microbiología en Brasil }\end{array}$ & Brasil \\
\hline PUS & 2009 & $\begin{array}{l}\text { Jurberg C, Verjovsky } \\
\text { M, De Oliveira D., } \\
\text { Affonso-Mitidieri O. }\end{array}$ & Células madre en los medios brasileños & Brasil \\
\hline JCOM & 2010 & $\begin{array}{l}\text { F.N. da Silva Medei- } \\
\text { ros y Massarani }\end{array}$ & $\begin{array}{l}\text { Cobertura de la epidemia H1N1 en los } \\
\text { noticieros de televisión }\end{array}$ & Brasil \\
\hline PUS & 2010 & Delgado A. & $\begin{array}{l}\text { La difusión del activismo verde en el } \\
\text { paisaje brasileño }\end{array}$ & Brasil \\
\hline PUS & 2010 & Stekolschik G. et al. & $\begin{array}{l}\text { Comunicación de la ciencia y vocacio- } \\
\text { nes científicas }\end{array}$ & Argentina \\
\hline SC & 2010 & $\begin{array}{l}\text { Gordon, J. C., T. Dei- } \\
\text { nes y J. Havice }\end{array}$ & $\begin{array}{l}\text { Cobertura sobre el cambio climático en } \\
\text { el periódico Reforma }\end{array}$ & México \\
\hline JCOM & 2011 & $\begin{array}{l}\text { Carneiro, M. J.y T. da } \\
\text { Silva Rosa }\end{array}$ & $\begin{array}{l}\text { El conocimiento científico en las políti- } \\
\text { cas públicas ambientales }\end{array}$ & Brasil \\
\hline PUS & 2011 & Takahashi, B. & $\begin{array}{l}\text { Cobertura periodística del cambio } \\
\text { climático en la V Reunión de la ALCUE }\end{array}$ & Perú \\
\hline PUS & 2011 & $\begin{array}{l}\text { Macnaghten, P. y J. S. } \\
\text { Guivant }\end{array}$ & $\begin{array}{l}\text { Nanotecnología y compromiso público } \\
\text { en Brasil y Reino Unido }\end{array}$ & $\begin{array}{l}\text { Brasil y } \\
\text { Reino Unido }\end{array}$ \\
\hline PUS & 2011 & $\begin{array}{l}\text { Kreimer P., L.Levin y } \\
\text { P. Jensen }\end{array}$ & $\begin{array}{l}\text { Actividades y popularización de la } \\
\text { ciencia por científicos argentinos }\end{array}$ & Argentina \\
\hline PUS & 2011 & Bentley P. y S. Kyvik & $\begin{array}{l}\text { Académicos y comunicación de la cien- } \\
\text { cia: encuesta en } 13 \text { países }\end{array}$ & $\begin{array}{l}\text { México, } \\
\text { Argentina, } \\
\text { Brasil y otros }\end{array}$ \\
\hline JCOM & 2012 & $\begin{array}{l}\text { Ramalho, M.,C. Po- } \\
\text { lino y L. Massarani }\end{array}$ & $\begin{array}{l}\text { Cobertura de la ciencia en noticieros de } \\
\text { televisión }\end{array}$ & Brasil \\
\hline PUS & 2012 & $\begin{array}{l}\text { Hurtado C. y J. A. } \\
\text { López Cerezo }\end{array}$ & $\begin{array}{l}\text { Políticas dimensiones de la cultura } \\
\text { científica en Iberoamérica }\end{array}$ & Iberoamérica \\
\hline PUS & 2012 & Vogt, Carlos & $\begin{array}{l}\text { La espiral de la cultura científica y } \\
\text { el bienestar cultural en Brasil y Ibero } \\
\text { America }\end{array}$ & $\begin{array}{l}\text { Brasil e } \\
\text { Iberoamérica }\end{array}$ \\
\hline SC & 2012 & Biro, S. & $\begin{array}{l}\text { Astronomía por correspondencia 1927- } \\
1947\end{array}$ & México \\
\hline
\end{tabular}


Tabla 3: Continúa desde la página anterior.

\begin{tabular}{|c|c|c|c|c|}
\hline Revista & Año & Autores & Tema & Territorio \\
\hline SC & 2012 & Duque, R. et al. & $\begin{array}{l}\text { Internet y colaboración entre científicos } \\
\text { en Chile }\end{array}$ & Chile \\
\hline JCOM & 2013 & Ríos P. y A. Negrete & $\begin{array}{l}\text { Arte y ciencia: comunicación de la cien- } \\
\text { cia por medio de instalaciones }\end{array}$ & México \\
\hline PUS & 2013 & $\begin{array}{l}\text { Takahashi B. y M. } \\
\text { Meisner }\end{array}$ & $\begin{array}{l}\text { El cambio climático en periódicos pe- } \\
\text { ruanos }\end{array}$ & Perú \\
\hline SC & 2013 & $\begin{array}{l}\text { Zamith, R., J. Pinto y } \\
\text { M. E. Villar }\end{array}$ & $\begin{array}{l}\text { Cobertura del cambio climático en pe- } \\
\text { riódicos de Estados Unidos y Sudaméri- } \\
\text { ca }\end{array}$ & $\begin{array}{l}\text { Argentina, } \\
\text { Brasil y } \\
\text { Colombia }\end{array}$ \\
\hline JCOM & 2014 & $\begin{array}{l}\text { Castelfranchi, Y., L. } \\
\text { Massarani y M. Ra- } \\
\text { malho }\end{array}$ & $\begin{array}{l}\text { La ciencia en la cobertura de los noticie- } \\
\text { ros de TV en Brasil }\end{array}$ & Brasil \\
\hline SC & 2014 & Biro $S$. & $\begin{array}{l}\text { Uso de un eclipse como performance } \\
\text { científico }\end{array}$ & México \\
\hline $\mathrm{JCOM}$ & 2015 & $\begin{array}{l}\text { Carlétti C. y L. Mas- } \\
\text { sarani }\end{array}$ & $\begin{array}{l}\text { Museos y centros de ciencia. Mediado- } \\
\text { res entre la ciencia y el público en Brasil }\end{array}$ & Brasil \\
\hline $\mathrm{JCOM}$ & 2015 & Hurtado, D. & $\begin{array}{l}\text { La construcción de Argentina como una } \\
\text { nación nuclear }\end{array}$ & Argentina \\
\hline PUS & 2015 & $\begin{array}{l}\text { Sánchez Mora, M. } \\
\text { del C. et al. }\end{array}$ & $\begin{array}{l}\text { Pasado, presente y futuro d la comuni- } \\
\text { cación de la ciencia en México }\end{array}$ & México \\
\hline PUS & 2015 & $\begin{array}{l}\text { Peplow D. y S. Au- } \\
\text { gustine }\end{array}$ & $\begin{array}{l}\text { Programas de salud pública como ac- } \\
\text { ción social en Surinam }\end{array}$ & Surinam \\
\hline PUS & 2015 & Muriello, S. & $\begin{array}{l}\text { Estudio de públicos de una exhibición } \\
\text { paleontológica. }\end{array}$ & Argentina \\
\hline JCOM & 2016 & $\begin{array}{l}\text { Sánchez Mora, M. } \\
\text { del C. }\end{array}$ & $\begin{array}{l}\text { Hacia una taxonomía de la comunica- } \\
\text { ción pública de la ciencia }\end{array}$ & México \\
\hline JCOM & 2016 & $\begin{array}{l}\text { Silva M. y L. Simo- } \\
\text { nian }\end{array}$ & $\begin{array}{l}\text { Diálogo entre la publicidad y la susten- } \\
\text { tabilidad en el amazonas peruano y bra- } \\
\text { sileño }\end{array}$ & Perú y Brasil \\
\hline JCOM & 2016 & Massarani, L. et al. & $\begin{array}{l}\text { Estudios de posgrado en comunicación } \\
\text { de la ciencia en América Latina }\end{array}$ & $\begin{array}{l}\text { América } \\
\text { Latina }\end{array}$ \\
\hline PUS & 2016 & Ureta, S. & $\begin{array}{l}\text { La Conferencia del Consenso Ciuda- } \\
\text { dano viaja a Chile }\end{array}$ & Chile \\
\hline SC & 2016 & $\begin{array}{l}\text { Rosen C., G. Lars y K. } \\
\text { Froehlinch }\end{array}$ & $\begin{array}{l}\text { Un estudio comparativo de criterios en } \\
\text { el periodismo científico de Argentina, } \\
\text { Francia y Alemania }\end{array}$ & $\begin{array}{l}\text { Argentina, } \\
\text { Francia y } \\
\text { Alemania }\end{array}$ \\
\hline JCOM & 2017 & $\begin{array}{l}\text { Rocha J.N. y M. Ma- } \\
\text { randino }\end{array}$ & $\begin{array}{l}\text { Museos de ciencia móviles y su historia } \\
\text { en la CPC }\end{array}$ & Brasil \\
\hline JCOM & 2017 & $\begin{array}{l}\text { Froes da Fonseca } \\
\text { M.R. }\end{array}$ & $\begin{array}{l}\text { La ciencia recreativa y la populariza- } \\
\text { ción de la ciencia en México en el siglo } \\
\text { XIX }\end{array}$ & México \\
\hline JCOM & 2017 & $\begin{array}{l}\text { Carvalho C. , M. Cat- } \\
\text { hia y S. Marcondes }\end{array}$ & $\begin{array}{l}\text { La comunicación de la psiquiatría en la } \\
\text { prensa brasileña 1930-1940 }\end{array}$ & Brasil \\
\hline PUS & 2017 & Almeida J. et al. & El cine científico en Brasil & Brasil \\
\hline
\end{tabular}

De los 40 artículos publicados 29 están localizado en un solo territorio, mientras que los otros once se refieren a dos o más territorios y hay cuatro que se refieren a toda Iberoamérica. Los principales escenarios de estudio son Brasil con 17 estudios individuales o con otros países; México con siete y Argentina con cuatro, obedecen a una cierta lógica en términos de su participación en la generación de conocimiento, aunque no es tan abismal la diferencia como la que resultó en la investigación 
Aproximaciones a la investigación en divulgación de la ciencia en América Latina a partir de artículos académicos que reportó "entre otros aspectos, una alta concentración de las publicaciones provenientes de Brasil, seguido por Colombia, Argentina y México," [Massarani, Rocha y col., 2017, p. 13]. En el estudio de Lars y Joubert también se destaca la supremacía brasileña en América Latina. [Guenther y Joubert, 2017].

El gran ausente es Colombia que aparece sólo en el estudio de Zamith, Pinto y Villar [2012] que presenta un análisis comparativo del concepto del cambio climático en cuatro periódicos nacionales en Argentina, Brasil, Colombia y Estados Unidos. Otros países latinoamericanos que aparecieron en la muestra son Perú con dos estudios individuales y un comparativo con Brasil; dos de Chile de carácter individual y uno de Surinam. Por otra parte, no es novedad que los estudios generales sobre América Latina sean tan desiguales en cuanto a su contenido.

En cuanto a los autores podemos encontrar en el corpus de 40 artículos, 72 nombres distintos, lo que da un promedio casi dos autores por artículo. Hay dos investigaciones firmadas por cinco autores [Duque y col., 2012; Massarani, Aguirre y col., 2015] y el otro extremo son nueve artículos firmados por un solo autor. La hegemonía de Brasil y en menor medida de México se refleja en las autorías múltiples. La brasileña Luisa Massarani es la autora que más textos tiene en la muestra con cinco artículos. Le siguen Susana Biro (UNAM), Bruno Takahasi (Michigan), Ana María Sánchez Mora (UNAM) y Elaine Reynoso-Heines (UNAM) con dos trabajos cada uno. El resto son autores distintos, lo que da una idea de la amplitud de los autores que investigan la comunicación pública de la ciencia en América Latina. Otro dato significativo es la presencia de autores/tema que se repiten en dos textos. No quiero decir que sean los mismos artículos, sino que tratan las mismas temáticas, como Biro y la historia de la astronomía en México; Massarani los noticieros de televisión en Brasil y Takahashi la cobertura del medio ambiente en periódicos peruanos. También hay que decir que Luisa Massarani es la autora más prolífica en diversos tipos de estudios sobre comunicación de la ciencia en Brasil y en América Latina. En toda su trayectoria y desde la presidencia ejecutiva de la Red POP (2016-17), ha promovido muchas investigaciones en donde ella ha actuado como coordinadora y autora de textos particulares. Por último, hay que destacar que los artículos firmados por dos o más autores y ubicados en más de un país implican un proceso de interinstitucionalización muy sano en los tiempos de globalización en los que vivimos.

Para las temáticas de los artículos hice una clasificación con diez categorías para los artículos: Ciencia, tecnología y medios de comunicación, participación social, percepciones, historia, arte, discursos, políticas públicas y gobierno, salud, museos y centros de ciencia y comunicación pública de la ciencia. La Tabla 4 muestras los resultados temáticos ordenados de mayor a menor.

La suma es superior al número de artículos, porque algunos están clasificados en dos temas. De todos los artículos del corpus, la mayor frecuencia corresponde a la relación entre la ciencia y la tecnología con los medios de comunicación. No es novedad esta tendencia porque en los escasos estudios previos [Orozco, 2015b; Massarani, Rocha y col., 2017], los medios de comunicación han sido los protagonistas principales de la investigación en comunicación pública de la ciencia. 
Tabla 4. Frecuencia de los temas en los artículos.

\begin{tabular}{|l|r|}
\hline Tema & Frecuencia \\
\hline CT y medios de comunicación & 14 \\
Comunicación pública de la ciencia & 7 \\
CT e historia & 4 \\
Museos y centros de ciencia & 4 \\
CT y percepciones & 4 \\
CT y participación social & 4 \\
CT políticas públicas y gobierno & 3 \\
CT y discursos & 1 \\
CT y arte & 1 \\
CT y salud & 1 \\
\hline
\end{tabular}

Massarani escribió al respecto:

\begin{abstract}
Los artículos recolectados fueron agrupados de acuerdo a las categorías que ilustran los temas de investigación en divulgación de la ciencia. Entre ellas, la categoría Medios y ciencia es la que concentra el mayor número de artículos, conteniendo el equivalente a $31 \%$ del número total de los trabajos recolectados, seguida por la categoría Museos y centros de ciencias, con $20 \%$, y Divulgación de la ciencia en la escuela, con $14 \%$. [Massarani, Rocha y col., 2017, p. 27].
\end{abstract}

En el caso del estudio de las tendencias internacionales en las tres revistas citadas, escribí que: "Los estudios que relacionan la ciencia y tecnología con los medios de comunicación son los más abundantes. En los cuatro años se publicaron 94 de un total de 380; lo que significa una proporción de $24.7 \%$, esto es uno de cada cuatro artículos analiza el enfoque o la cobertura que los medios han dado a un tema de ciencia. [Orozco, 2015b, p. 43]

El dato de que en los tres estudios disponibles, las investigaciones que relacionan la comunicación de la ciencia y la tecnología con los medios de comunicación es la más frecuente se podría explicar por varias razones; a) la comunicación pública de la ciencia surgió de la articulación entre los estudios sociales de la ciencia y las investigaciones sobre la comunicación de masas; $b$ ) en la comunicación como disciplina, también los estudios de los medios ocupa un lugar destacado y c) en términos de simple pragmatismo, los estudios de cobertura o análisis de contenido se pueden hacer con pocos recursos.

Una clasificación de segundo nivel en el apartado de los medios y la CPC, lleva a establecer que la mitad - siete de los catorce estudios — se refieren a periódicos; tres a televisión, uno a cine y otro a publicidad. Tampoco en esta subclasificación hay novedad; los diarios y la televisión son los medios más estudiados desde la comunicación. Lo novedoso en esta subclasificación es la investigación de Silva y Simonian [2016] sobre el diálogo entre la publicidad y la sustentabilidad en la región del Amazonas. La relación entre estos dos conceptos y sus concreciones, prácticamente no se había abordado.

El medio ambiente en general y el cambio climático en particular - al igual que en los estudios internacionales [Orozco, 2015b] — es el objeto de estudio preferido en 
las investigaciones entre comunicación pública de la ciencia y los medios de comunicación. De los 40 estudios, ocho, (20 por ciento) están orientados a las temáticas ambientales.

La comunicación pública de la ciencia (CPC), entendida en este ejercicio de tendencias de investigación, como estudio de carácter general y que busca contribuir al estado del conocimiento más de tipo conceptual, aunque hay algunos reportes de estudios empíricos, está representada por siete investigaciones. Estudios sobre la influencia de la CPC en las vocaciones científicas en Argentina [Stekolschik y col., 2010]; otro sobre la participación de los investigadores en tareas de divulgación también en Argentina [Kreimer, Levin y Jensen, 2011]; uno más sobre investigadores científicos y CPC, pero éste comparativo entre 13 naciones [Bentley y Kyvik, 2011]; otra sobre el pasado, presente y futuro de la CPC en México [Sánchez-Mora y col., 2015]; otro centrado en una taxonomía de las actividades de la divulgación - el estudio más teórico del corpus - [Carmen Sánchez-Mora, 2016], y otro sobre diplomados y estudios de posgrado en la comunicación pública de la ciencia en América Latina [Massarani, Reynoso-Haynes y col., 2016]. Los estudios históricos sobre algún aspecto, por ejemplo, la astronomía, de la ciencia; los museos y centros de ciencia y las percepciones ocupan los espacios medio de la tabla de concentración con tres o cuatro estudios. La participación social, las políticas públicas, los discursos, el arte y la salud, todas con relación a la ciencia y la tecnología son los temas con menor participación en la tabla. Es de destacar que el tema de la comunicación del riesgo enfocado a la salud aparece solo en un estudio mientras que en los temas de investigación de los países desarrollados ocupa un lugar destacado, sobre todo en las percepciones del riesgo en la salud humana de productos genéticamente modificados, nanotecnología aplicada a la salud o medicina genómica [Orozco, 2015b]. Hay también un estudio que analiza comparativamente la percepción de la nanotecnología en entre los ciudadanos de Brasil con relación a los del Reino Unido [Macnaghten y Guivant, 2011]. Volviendo al tema de salud es paradójico que una región como América Latina con tantas carencias en materia de salud pública se investigue mucho menos sobre la relación entre comunicación pública de la ciencia y salud que en los países con mejores coberturas de salud como los europeos. El estudio de Peplow y Augustine [2015] sobre las políticas de salud pública en Surinam es la excepción en esta tendencia.

Los métodos utilizados en las investigaciones reflejan con claridad el origen y la práctica actual interdisciplinaria de la comunicación pública de la ciencia. En los 40 estudios del corpus, hay 15 métodos distintos provenientes de la historia, la sociología, la antropología, la comunicación e incluso de metodologías experimentales.

Los métodos utilizados son diversos. Con excepción de uno

[Carmen Sánchez-Mora, 2016] que es conceptual, el resto de los artículos tienen un componente empírico, pero en cuanto a métodos particulares hay una mayor proporción de análisis de contenido o estudios comparativos, pero también encontramos etnografía [Delgado, 2010], análisis epistolar [Biro, 2012; Biro, 2014], uso de encuestas [Bentley y Kyvik, 2011; Duque y col., 2012; M. C. Hurtado y López Cerezo, 2012; Kreimer, Levin y Jensen, 2011; Stekolschik y col., 2010], entrevistas [Macnaghten y Guivant, 2011] y hasta el método experimental [Rios y Negrete, 2013]. 
En la Tabla 5 aparecen los métodos utilizados en las investigaciones del corpus. La suma es superior a los 40 reportes, porque hay investigaciones [por ejemplo Bentley y Kyvik, 2011] que usaron más de un método.

Tabla 5. Frecuencia de los métodos de los artículos.

\begin{tabular}{|l|r|}
\hline Método & Frecuencia \\
\hline Estudio de caso & 9 \\
Estudio comparativo & 8 \\
Análisis de contenido & 7 \\
Encuestas & 5 \\
Estudio histórico & 3 \\
Estudio experimental & 1 \\
Estudio etnográfico & 1 \\
Análisis de correspondencia & 1 \\
Análisis de red & 1 \\
Evolución del campo & 1 \\
Estudio de públicos & 1 \\
Mapeo & 1 \\
Estudio taxonómico & 1 \\
Evolución del impacto & 1 \\
Estudios CTS & 1 \\
\hline
\end{tabular}

Discusión y conclusiones
La comunicación del riesgo, en su vertiente del medio ambiente es la principal temática que se aborda en estas investigaciones empíricas. No es sorpresivo porque también en un estudio similar [Orozco, 2015b], pero con todos los artículos de investigación publicados en las tres revistas entre 2010 y 2012, la temática del medio ambiente - en particular, el cambio climático - fue la más estudiada y el enfoque metodológico más utilizado fue el análisis de contenido sobre la cobertura de algún medio con relación a esta problemática. En el corpus para esta investigación encontramos ocho textos (de un total de 40), da Carneiro y da Silva Rosa [2011], da Silva Medeiros y Massarani [2010], Delgado [2010], Gordon, Deines y Havice [2010], Ramalho, Polino y Massarani [2012], Takahashi [2011], Takahashi y Meisner [2013] y Zamith, Pinto y Villar [2012] en los que se estudia la cobertura del cambio climático en periódicos de México, Perú, Brasil, Argentina o Colombia. Este resultado puede ser consecuencia de la mediatización de la ciencia, como fue señalado por Polino y Castelfranchi: "los medios de comunicación han jugado un rol decisivo en la amplificación de los riesgos asociados con el desarrollo de la ciencia y la tecnología. Esto ha sucedido aparentemente en Estados Unidos y Europa, pero también en América Latina". [Polino y Castelfranchi, 2012, p. 11].

Podemos decir que América Latina ha sido más un escenario de estudio, en particular para temas mundiales como el calentamiento global, que una región productora de conocimiento original en la comunicación pública de la ciencia. La incipiente investigación que se está haciendo responde más a la lógica de conocimiento e intereses de conocimiento de los países con una masa crítica de científicos y comunicadores profesionales, como Estados Unidos, Reino Unido o Europa que a las necesidades particulares de nuestros países. De los 40 artículos incluidos en el corpus, casi una quinta parte (7) son investigaciones hechas desde 
Estados Unidos o el Reino Unido o hacen comparaciones con naciones desarrolladas. Estos artículos son: Bentley y Kyvik [2011], Gordon, Deines y Havice [2010], Macnaghten y Guivant [2011], Takahashi [2011], Takahashi y Meisner [2013], Zamith, Pinto y Villar [2012], Peplow y Augustine [2015] y Rosen, Guenther y Froehlich [2016].

Otro aspecto significativo son los artículos colaborativos entre autores de dos o más países que estudian temas específicos y los comparan entre ellos. En el corpus de 40 artículos publicados, siete - uno de cada cinco - pertenecen a esta categoría.

Por último, no debemos de ignorar el fenómeno de la globalización de la producción y circulación del conocimiento, que también se expresa en la comunicación pública de la ciencia. Daniel Mato, un estudioso de los procesos socioculturales en América Latina argumenta:

\begin{abstract}
Hay algunos aspectos particulares de los procesos de globalización contemporáneos que pueden resultar especialmente significativos para nuestra reflexión. (...) La creciente importancia de redes de relaciones transnacionales en la producción de ideas y programas de acción social y política significativos. Estas redes pueden estar conformadas por actores sociales esparcidos por el mundo (no me refiero a su mera existencia en Internet, que es sólo un medio) pero frecuentemente son organizadas y sostenidas por actores localizados en Estados Unidos y/o en unos pocos países de Europa Occidental, quienes de este modo tienen ciertas ventajas en el planteamiento de las ideas en torno a las cuales se articulan, así como en la proposición de sus programas de acción. [Mato, 2002, p. 23].
\end{abstract}

Esta reflexión de Mato es pertinente para el caso de la investigación académica en comunicación pública de la ciencia en América Latina en donde encontramos una vinculación creciente en investigadores de la región (el ejemplo más claro está en los libros publicados por la Red Pop), pero al mismo tiempo, no parecen ser muchas las evidencias de trabajos conjuntos entre investigadores latinoamericanos con sus homólogos de otros países, especialmente Estados Unidos y Europa. Y lo escribo no como afirmación categórica, porque en el diseño de esta investigación no se incluyó la nacionalidad o el lugar de trabajo de los investigadores como variable de estudio. Este reto de internacionalización en conjunto con colegas de países con mayor producción científica debe estar en la agenda de la investigación latinoamericana en comunicación pública de la ciencia. Sería una forma de reducir la globalización dispareja que vivimos.

\title{
Referencias
}

BENTLEY, P. y KYVIK, S. (2011). 'Academic staff and public communication: A survey of popular science publishing across 13 countries'. Public Understanding of Science 20 (1), págs. 48-63. https://doi .org/10.1177/0963662510384461.

BIRO, S. (2012). 'Astronomy by correspondence: a study of the appropriation of science by the Mexican public (1927-1947)'. Science Communication 34 (6), págs. 803-819. https://doi.org/10.1177/1075547012438466.

- (2014). 'Natural wonders and scientific performance. A Mexican eclipse and its uses'. Science Communication 36 (6), págs. 735-753. https://doi.org/10.1177/1075547014554960. 
BUCCHI, M. y TRENCH, B., eds. (2014). Routledge Handbook of Public

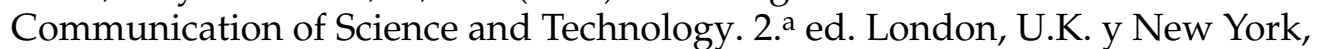
U.S.A.: Routledge. https://doi .org/10.4324/9780203483794.

CARLÉTTI, C. y MASSARANI, L. (2015). 'Explainers of science centres and museums: a study on these stakeholders in the mediation between science and the public in Brazil'. JCOM 14 (02), A01.

URL: https://jcom.sissa.it/archive/14/02/JCOM_1402_2015_A01.

CARMEN SÁNCHEZ-MORA, M. del (2016). ‘Towards a taxonomy for public communication of science activities'. JCOM 15 (02), Y01. https://doi.org/10.22323/2.15020401.

CARNEIRO, M. J. y DA SILVA ROSA, T. (2011). 'The use of scientific knowledge in the decision making process of environmental public policies in Brazil'. JCOM 10 (01), A03. https://doi.org/10.22323/2.10010203.

CARVALHO JACOBUCCI, D. F. y JACOBUCCI, G. B. (2009). 'Opening the test tube: what do we know about research on science communication and the teaching of microbiology in Brazil?' JCOM 08 (02), A02. https://doi.org/10.22323/2.08020202.

CARVALHO, C., MATHIAS, C. y MARCONDES, S. (2017). 'The communication of psychiatry in Brazilian press (1930-1940)'. JCOM 16 (03), A13. https://doi.org/10.22323/2.16030213.

CASTELFRANCHI, Y., MASSARANI, L. y RAMALHO, M. (2014). 'War, anxiety, optimism and triumph: a study on science in the main Brazilian TV news'. JCOM 13 (3), A01. URL: https://jcom.sissa.it/archive/13/03/JCOM_1303_2014_A01.

DA SILVA MEDEIROS, F. N. y MASSARANI, L. (2010). 'Pandemic on the air: a case study on the coverage of new influenza A/H1N1 by Brazilian prime time TV news'. JCOM 09 (03), A03. https://doi.org/10.22323/2.09030203.

DE ALMEIDA, J., DA SILVA, C. I., SUPPIA, A. y STALBAUM, B. (2016). 'Passages on Brazilian scientific cinema'. Public Understanding of Science 26 (5), págs. 579-595. https://doi.org/10.1177/0963662516683638.

DELGADO, A. (2010). 'Activist trust: the diffusion of green expertise in a Brazilian landscape'. Public Understanding of Science 19 (5), págs. 562-577. https://doi.org/10.1177/0963662508098578.

DUQUE, R. B., MILLER, B. P., BARRIGA, O., SHRUM, W. y HENRÍQUEZ, G. (2012). 'Is Internet use associated with reporting fewer problems in collaboration? Evidence from the scientific community in Chile'. Science Communication 34 (5), págs. 642-678. https://doi.org/10.1177/1075547011432364.

FERNÁNDEZ POLPUCH, E., BELLO, A. y MASSARANI, L., eds. (2016). Políticas públicas e instrumentos para el desarrollo de la cultura científica en América Latina. Montevideo, Uruguay: Laboratorio Tecnológico del Uruguay, Red POP, UNESCO.

FIRER, M. (2008). ‘Oficina Desafio — Challenging creativity'. JCOM 07 (03), A02. https://doi.org/10.22323/2.07030202.

FRÓES DA FONSECA, M. R. (2017). 'La ciencia recreativa and the popularisation of science in Mexico in the 19th century'. JCOM 16 (03), A07. https://doi.org/10.22323/2.16030207.

FUENTES NAVARRO, R. (1998). La emergencia de campo académico. Continuidad utópica y estructuración científica de la investigación de la comunicación en México. Guadalajara, Mexico.

FUENTES NAVARRO, R. y OROZCO, C. E. (1997). Maestría en comunicación con especialidad en difusión de la ciencia y la cultura. Diseño curricular y estructura del plan de estudios. Documento interno. Mexico. 
GORDON, J. C., DEINES, T. y HAVICE, J. (2010). 'Global warming coverage in the media: trends in a Mexico city newspaper'. Science Communication 32 (2), págs. 143-170. https://doi.org/10.1177/1075547009340336.

GREGORY, J. y MILLER, S. (1998). Science in public: communication, culture, and credibility. London, U.K. y New York, U.S.A.: Plenum.

GUENTHER, L. y JOUBERT, M. (2017). 'Science communication as a field of research: identifying trends, challenges and gaps by analysing research papers'. JCOM 16 (02), A02. https://doi .org/10.22323/2.16020202.

HERRERA, A. O. (2015). Ciencia y política en América Latina. (Original en 1970). Buenos Aires, Argentina: Biblioteca Nacional.

HURTADO, D. (2015). 'Semi-periphery and capital-intensive advanced technologies: the construction of Argentina as a nuclear proliferation country'. JCOM 14 (02), A05. https://doi.org/10.22323/2.14020205.

HURTADO, M. C. y LÓPEZ CEREZO, J. A. (2012). 'Political dimensions of scientific culture: highlights from the Ibero-American survey on the social perception of science and scientific culture'. Public Understanding of Science 21 (3), págs. 369-384. https://doi .org/10.1177/0963662510373871.

JURBERG, C., VERJOVSKY, M., DE OLIVEIRA CARDOSO MACHADO, G. y AFFONSO-MITIDIERI, O. R. (2009). 'Embryonic stem cell: a climax in the reign of the Brazilian media'. Public Understanding of Science 18 (6), págs. 719-729. https://doi.org/10.1177/0963662509335457.

KREIMER, P., LEVIN, L. y JENSEN, P. (2011). 'Popularization by Argentine researchers: the activities and motivations of CONICET scientists'. Public Understanding of Science 20 (1), págs. 37-47. https://doi.org/10.1177/0963662510383924.

LEMARCHAND, A., ed. (2010). Sistemas nacionales de ciencia, tecnología e innovación en América Latina. Montevideo, Uruguay: UNESCO, Oficina Regional de la Ciencia para América Latina y el Caribe.

MACNAGHTEN, P. y GUIVANT, J. S. (2011). 'Converging citizens? Nanotechnology and the political imaginary of public engagement in Brazil and the United Kingdom'. Public Understanding of Science 20 (2), págs. 207-220. https://doi.org/10.1177/0963662510379084.

MASSARANI, L. (2015). 'Voices from other lands'. Public Understanding of Science 24 (1), págs. 2-5. https://doi .org/10.1177/0963662514563888.

MASSARANI, L., AGUIRRE, C., PEDERSOLI, C., E., R. y LINDEGAARD, L. M. (2015). 'RedPOP: 25 años de Red en Comunicación de la Ciencia en América Latina'. JCOM 14 (03), Y06. URL: http://jcom.sissa.it/sites/default/file s/documents/JCOM_1403_2015_Y06.

MASSARANI, L., REYNOSO-HAYNES, E., MURRIELLO, S. y CASTILLO, A. (2016). 'Science communication postgraduate studies in Latin America: a map and some food for thought'. JCOM 15 (05), A03. https://doi.org/10.22323/2.15050203.

MASSARANI, L., ROCHA, M., PEDERSOLI, C., ALMEIDA, C., AMORIM, L., CAMBRE, M., NEPOTE, A. C., NOBERTO ROCHA, J., AGUIRRE, C., GONÇALVEZ, J. C., CORDIOLI, L. y FERREIRA, F., eds. (2017). Aproximaciones a la investigación en divulgación de la ciencia en América Latina a partir de sus artículos académicos. $1 .^{\text {a }}$ ed. Rio de Janeiro, Brazil: Fiocruz, Casa de Oswaldo Cruz. URL: http://bit.ly/2vMYMgX.

MATO, D. (2002). 'Estudios y otras prácticas intelectuales latinoamericanas en cultura y poder'. En: Estudios y otras prácticas intelectuales latinoamericanas en cultura y poder. Ed. por MATO, D. Caracas, Venezuela: Universidad Central de Venezuela y Consejo Latinoamericano de Ciencias Sociales (CLACSO), págs. 21-45. 
MURRIELLO, S. (2015). 'The palaeontological exhibition: a venue for dialogue'. Public Understanding of Science 24 (1), págs. 86-95. https://doi.org/10.1177/0963662514555452.

NORBERTO ROCHA, J. y MARANDINO, M. (2017). 'Mobile science museums and centres and their history in the public communication of science'. JCOM 16 (03), A04. https://doi.org/10.22323/2.16030204.

OEA. ORGANIZACIÓN DE ESTADOS AMERICANOS (1967). Declaración de Punta del Este. Punta del Este, Uruguay: Organización de Estados Americanos.

OROZCO, C. E. (2015a). ‘Las tendencias de la investigación de la comunicación pública de la ciencia en América Latina. Un estudio en tres revistas académicas internacionales (2010-2014)'. En: XV Encuentro Latinoamericano de Facultades de Comunicación Social FELAFACS. Medellín, Colombia.

- (2015b). 'Tendencias de la investigación académica internacional en la comunicación pública de la ciencia'. En: De la academia al espacio público. Comunicar ciencia en México. Ed. por HERRERA LIMA, S., OROZCO, C. E. y QUIJANO, E. Vol. 2. Guadalajara, Mexico: ITESO.

PASQUALI, A. (1970). Comprender la comunicación. Caracas, Venezuela: Monte Ávila.

PATIÑO, M. L., PADILLA, J. y MASSARANI, L. (2017). Diagnóstico de la Divulgación de la Ciencia en América Latina: Una mirada a la práctica en el campo. 1. ${ }^{\mathrm{a}}$ ed. Ciudad de México, México: Fibonacci e RedPOP. URL: http://www.redpop.org/wp-content/uploads/2017/06/Diagnostico-d ivulgacion-ciencia_web.pdf.

PEPLOW, D. y AUGUSTINE, S. (2015). 'Public health programs as surrogates for social action in Suriname, South America'. Public Understanding of Science 24 (1), págs. 53-68. https://doi.org/10.1177/0963662513513397.

PIOLLI, A. L. y CONCEIÇÃO DA COSTA, M. (2008). 'Public participation and rural management of Brazilian waters: an alternative to the deficit model'. JCOM 07 (04), A01. https://doi.org/10.22323/2.07040201.

POLINO, C. y CASTELFRANCHI, Y. (2012). 'The 'communicative turn' in contemporary techno-science: latin american approaches and global tendencies'. En: Science communication in the world: practices, theories and trends. Ed. por SCHIELE, B., CLAESSENS, M. y SHI, S. Dordrecht, The Netherlands: Springer, págs. 3-17. https://doi.org/10.1007/978-94-007-4279-6_1.

RAMALHO, M., POLINO, C. y MASSARANI, L. (2012). 'From the laboratory to prime time: science coverage in the main Brazilian TV newscast'. JCOM 11 (02), A02. https://doi.org/10.22323/2.11020202.

REIS, R. (2008). 'How Brazilian and North American Newspapers Frame the Stem Cell Research Debate'. Science Communication 29 (3), págs. 316-334.

RIOS, P. y NEGRETE, A. (2013). 'The object of art in science: science communication via art installation'. JCOM 12 (03), A04. https://doi.org/10.22323/2.12030204.

ROSEN, C., GUENTHER, L. y FROEHLICH, K. (2016). 'The question of newsworthiness. A cross-comparision about science journalists' selection criteria in Argentine, France and Germany'. Science Communication 38 (3), págs. 328-355. https://doi .org/10.1177/1075547016645585.

SÁNCHEZ-MORA, C., REYNOSO-HAYNES, E., SÁNCHEZ MORA, A. M. y TAGÜEÑA, J. (2015). 'Public communication of science in Mexico: past, present and future of a profession'. Public Understanding of Science 24 (1), págs. 38-52. https://doi .org/10 .1177/0963662514527204. PMID: 24789844. 
SILVA, M. y SIMONIAN, L. (2016). 'How advertising and sustainability dialog in Pan-Amazonia: the perspective of advertising professionals in Peru and Brazil'. JCOM 15 (05), A01. https://doi.org/10.22323/2.15050201.

STEKOLSCHIK, G., DRAGHI, C., ADASZKO, D. y GALLARDO, S. (2010). ‘Does the public communication of science influence scientific vocation? Results of a national survey'. Public Understanding of Science 19 (5), págs. 625-637. https://doi.org/10.1177/0963662509335458.

TAIT LIMA, M., FELIX DAS NEVES, E. y DAGNINO, R. (2008). 'Popularization of science in Brazil: getting onto the public agenda, but how?' JCOM 07 (04), A02. https://doi.org/10.22323/2.07040202.

TAKAHASHI, B. (2011). 'Framing and sources: a study of mass media coverage of climate change in Peru during the V ALCUE'. Public Understanding of Science 20 (4), págs. 543-557. https://doi.org/10.1177/0963662509356502.

TAKAHASHI, B. y MEISNER, M. (2013). 'Climate change in Peruvian newspapers: the role of foreign voices in a context of vulnerability'. Public Understanding of Science 22 (4), págs. 427-442. https : //doi .org/10.1177/0963662511431204.

TRABULSE, E. (1983). Historia de la ciencia en México. 4 tomos. México: D.F. Consejo Nacional de Ciencia y Tecnología y Fondo de Cultura Económica.

TRENCH, B. y BUCCHI, M. (2010). 'Science communication, an emerging discipline'. JCOM 09 (03), C03. https : //doi .org/10.22323/2.09030303.

URETA, S. (2016). 'A failed platform: the citizen consensus conference travels to Chile'. Public Understanding of Science 25 (4), págs. 499-511. https://doi.org/10.1177/0963662514561940.

VENEU, F., AMORIM, L. H. y MASSARANI, L. (2008). 'Science journalism in Latin America: how the scientific information from a scientific source is accommodated into a journalistic story'. JCOM 7 (1), A03. URL: https://jcom.sissa.it/archive/07/01/Jcom0701\%282008\%29A03.

VOGT, C. (2012). 'The spiral of scientific culture and cultural well-being: Brazil and Ibero-America'. Public Understanding of Science 21 (1), págs. 4-16. https://doi.org/10.1177/0963662511420410.

ZAMITH, R., PINTO, J. y VILLAR, M. E. (2012). 'Constructing climate change in the Americas: an analysis of news coverage in U.S. and South American newspapers'. Science Communication 35 (3), págs. 334-357. https://doi.org/10.1177/1075547012457470.

Carlos Enrique Orozco se ha desempeñado profesionalmente entre el periodismo y la academia. Fue fundador del diario Siglo 21 de Guadalajara, en donde publicó una columna semanalmente sobre ciencia y cultura. Fue coordinador fundador de la Maestría en Comunicación de la Ciencia y la Cultura del ITESO, misma que coordina nuevamente. Ha publicado artículos de divulgación y textos académicos sobre la comunicación pública de la ciencia y es co-editor de la serie de libros "Comunicar Ciencia en México". E-mail: carlose@iteso.mx.

\section{Cómo citar}

Orozco, C. E. (2018). ‘Diez años de investigación de la comunicación pública de la ciencia en y desde América Latina. Un estudio en tres revistas académicas (2008-2017)'. JCOM - América Latina 01 (01), A02. https://doi.org/10.22323/3.01010202. 